\title{
Submitted: \\ 22.04.2020 \\ Accepted: \\ Evaluation of benign parotid gland tumors with superb microvascular imaging and shear wave elastography
}

21.06.2020

Published:

28.09.2020

\author{
Keywords \\ superb microvascular \\ imaging, \\ shear wave \\ elastography, \\ parotid gland, \\ tumor
}

\author{
Hakan Cebeci ${ }^{1}$, Mehmet Öztürk ${ }^{1}$, Mehmet Sedat Durmaz¹, \\ Abidin Kılınçer ${ }^{1}$, Ömer Erdur², Bahar Çolpan² \\ ${ }^{1}$ Department of Radiology, Selcuk University, Faculty of Medicine, Konya, Turkey \\ ${ }^{2}$ Department of Otorhinolaryngology, Selcuk University, Faculty of Medicine, Konya, Turkey \\ Correspondence: Hakan Cebeci, MD, Assistant Professor, Selcuk University Faculty of Medicine, \\ Selcuklu, Konya, Turkey, 42130; tel. +90332 2415000,e-mail: hcebeci16@gmail.com
}

DOI: $10.15557 / \mathrm{JoU} .2020 .0031$

\begin{abstract}
Aim of the study: This study aimed to examine the role of superb microvascular imaging and shear wave elastography for the pre-surgical evaluation of common parotid tumors. Material and methods: This single-center prospective study included 37 patients with parotid gland lesions. After institutional review board approval, grayscale, shear wave elastography and superb microvascular imaging ultrasound examinations were performed prior to biopsy or operation. The diagnosis of the lesions was based on cytological/pathological evaluation after the ultrasound examinations. Pleomorphic adenomas and Warthin tumors were compared using the Mann-Whitney U test. A receiver operating characteristic curve analysis was performed to obtain a cut-off value. A multivariate regression analysis was carried out. Results: The mean age of the patients (11 female, 26 male) was $48.2 \pm 18$. The shear wave elastography parameters of the lesions were not significantly different between pleomorphic adenomas and Warthin tumors, while the vascular index obtained by using superb microvascular imaging was significantly different $(p=0.012)$. The mean vascular index was $2.9 \pm 3.1$ in pleomorphic adenomas, and $9.5 \pm 9.5$ in Warthin tumors. A cut-off value of 4.05 for the vascular index discriminated pleomorphic adenoma and Warthin tumors with $68 \%$ sensitivity and $72 \%$ specificity (the area under the curve was 0.768 ). Conclusion: Superb microvascular imaging is a novel ultrasound imaging technique which is useful for the discrimination of pleomorphic adenomas and Warthin tumors.
\end{abstract}

\section{Introduction}

The pre-surgical diagnosis of salivary gland tumors consists of challenges despite advances in imaging techniques. The surgical treatment of malignant lesions requires more radical approaches, thus some patients need to undergo a second surgery when the histology of the surgical specimen differs from the pre-surgical diagnosis ${ }^{(1)}$.

Ultrasound (US) is a commonly used diagnostic imaging technique for evaluating parotid glands non-invasively. US shows the precise location of tumoral lesions, differentiates solid tumors from cystic lesions with avoiding ionizing radiation with lower costs. Although grayscale, color and power Doppler US show sonographic features of some specific diagnosis, it is challenging in many cases to differentiate between benign and malignant tumors. Also, it is difficult to make a distinction between pleomorphic adenomas and Warthin tumors, which are the most common parotid gland tumors ${ }^{(2,3)}$.

Shear wave elastography (SWE) is an imaging technique for measuring elasticity. It provides data regarding tissue stiffness using focused pulses of ultrasound (push pulses) to stress tissues, and obtains quantitative data about tissue stiffness. This imaging technique allows measurements of the propagation speed of shear waves within the tissue to locally quantify its stiffness in kilopascals or meters per second. SWE is a particularly common sonography technique in superficial organs. Previous reports exist in the literature reporting the role of SWE in parotid tumors, and also thyroid and breast lesions ${ }^{(2,4-9)}$. 


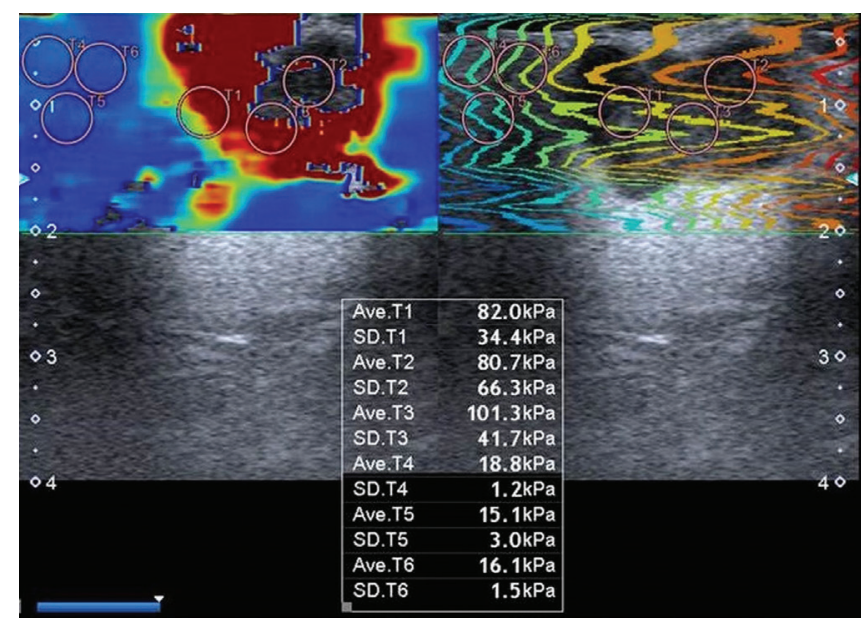

Fig. 1. Three different measurements of elasticity and shear wave velocity in a single transverse plane from the tumor core and normal appearing parotid gland



Fig. 2. Measurement of the vascular index during cSMI examination by lining tumor borders

Superb micro-vascular imaging (SMI) is a novel Doppler ultrasound technique designed to overcome the limitations of conventional Doppler US techniques. SMI is used to determine microvascular blood flow. SMI decreases signals produced by movement, and reveals low-velocity blood flow. As a result, both high-velocity and low-velocity blood flow signals establish an image in the SMI technique ${ }^{(10)}$. SMI allows the visualization of small vascular signals with a high image resolution and high frame rate (>50 fps). SMI includes two different modes: color SMI (cSMI) and monochrome SMI (mSMI). The cSMI mode simultaneously displays conventional grayscale ultrasound (US) with color-encoded Doppler signals. The mSMI mode suppresses the background signals and focuses only on the vascular signals, thus increasing the visibility of vascular structures $^{(11,12)}$. To our knowledge, there is only one previous study addressing the use of SMI in parotid gland tumors ${ }^{(13)}$.

The present study aimed to describe our preliminary experience with using cSMI and SWE to evaluate focal parotid gland tumors. Conventional sonographic criteria and color
Doppler US were not evaluated in the study, as they are extensively documented in the medical literature.

\section{Materials and methods}

PatientsThis prospective study was conducted between January 2018 and July 2019. An institutional review board approved the study, and written informed consent was obtained before performing the US examinations. All consecutive patients with histologically or cytologically proven parotid gland tumors admitted to a otolaryngology outpatient clinic in the study period were included. Ultrasound, SWE and SMI characteristics were compared with the pathological diagnosis. The lesions were grouped into benign and malignant tumors. In the benign tumor group, the lesions were sub-grouped as pleomorphic adenomas and Warthin tumors. The pathological results were obtained via surgical excision, tru-cut biopsy, and/or fine needle aspiration biopsy.

\section{US examinations}

All US examinations were performed with same US equipment (Aplio 500, Toshiba Medical System Corporation, Tokyo, Japan), with a high-frequency (4-14 MHz) linear array transducer, and by a single radiologist (MO; 12-yearexperience in US, 6-year experience in SWE, and 3-year experience in SMI). In all the patients, grayscale US, SWE and SMI US examinations were performed before surgery and/or biopsy, in the supine position with the head turned to the opposite side. Detailed grayscale US examinations were performed, and the location, size, cystic and solid parts of tumor were assessed.

After the grayscale US examination, SWE examination was performed. In the split-screen mode, 2D-SWE map (left side) and quality mode (right side) were examined. The measurements were conducted when the lines were parallel and smooth in quality mode. After stabilization for $5 \mathrm{~s}$, the SWE images were frozen and saved. The elasticity range was set to $0-50 \mathrm{kPa}$, and the velocity range was set to $0-8 \mathrm{~m} / \mathrm{s}$ on the standardized setting. Then, a 5-10 mm diameter "region of interest (ROI)" was used to take measurements at three different points. Three different ROIs were placed to both tumor core and the normal appearing parotid gland parenchyma. If the tumor consisted of cystic parts, the ROIs were placed to the solid parts of the lesion. Fig. 1 demonstrates the manner of ROI placement to the tumoral lesion and normal parotid gland tissue. When the tumoral lesion covered the entire parotid gland, then measurements of the other side of the parotid gland were performed. The mean of the three different measured ROIs was calculated. The elasticity and shear wave velocity values were recorded. The relative elasticity and shear wave velocity values were calculated through dividing the lesion values by the normal parotid gland parenchymal values. During the examination, a sufficient amount of US gel was used, no pressure was applied to the probe, and care was taken to make sure that the operator's hand was not moving.

The cSMI examination was performed after the SWE, showing blood flow in a color display. For the sufficient 




Fig. 3. Shear wave elastography (A) and color superb microvascular imaging (B) examinations of a 29-year-old female patient having a pathologically proven pleomorphic adenoma on the left parotid gland. There was no vascular signal in the tumor core

elimination of background color signals, the color gain was automatically set to $40 \mathrm{~dB}$. In all cases, a window was drawn manually lining tumor borders, and the vascular index of the tumor was calculated. Three different measurements were taken, and the highest value of the vascular index was adopted for evaluation. The measurement of the vascular index by c-SMI is demonstrated in Fig. 2.

\section{Statistical analysis}

SPSS 21.0 (IBM Corporation, Armonk, New York, USA) was used for the analysis of variables. Descriptive statistics were expressed as the mean \pm SD. Categorical variables were tested by Fisher's exact test. The Mann-Whitney U test was used for the comparison of stiffness, velocity and VI values between pleomorphic adenomas and Warthin tumors. The Spearman correlation analysis was used to evaluate the relationship between the VI values, the lesion area in $\mathrm{mm}^{2}$, and stiffness and velocity values. The statistical significance level was defined as 0.05 . A receiver operating characteristic (ROC) curve analysis was performed to determine the best cut-off value for the vascular index values in pleomorphic adenoma and Warthin tumors.

\section{Results}

The patients were between 6 and 84 years old (mean age: $48.24 \pm 18.08)$. Thirty-seven parotid gland tumors in 37 patients (26 male, 11 female) were included. All parotid lesions were unilateral. The cytological/pathological diagnoses of the lesions were as follows: 14 pleomorphic adenomas, 16 Warthin tumors, 1 indistinct benign tumor, 1 benign lymphoid tissue, 1 adenoid cystic carcinoma, 1 secretory carcinoma, 2 non-differentiated carcinomas, and 1 inflammatory myofibroblastic tumor. The tissue diagnosis was made by surgical excision in 25 tumors, US guided tru-cut biopsy in 5, and fine-needle aspiration biopsy in 7 tumors. The descriptive analysis including age, gender, elasticity (in kilopascals), shear wave velocity (in meters per second) values of the tumor and normal parotid gland tissue, the area of the measured lesion and the vascular index of the tumor obtained by cSMI is shown in Tab. 1 and Tab. 2 .

In SWE measurements, the mean elasticity of normal parotid gland parenchyma was $26.8 \pm 21.6$, and the mean shear wave velocity of normal parenchyma was $2.7 \pm 1.1$. The mean elasticity and shear wave velocity of benign lesions were higher in the malignant group. The vascular index was lower in the benign group than in the malignant group. The mean vascular index was $6.2 \pm 7.5$ in benign lesions, and $4.7 \pm 4.5$ in malignant lesions. However, because of the low number of lesions in the malignant group, we did not perform a statistical comparison.

A comparison of pleomorphic adenomas and Warthin tumors revealed no significant differences in terms of SWE parameters. The vascular index was significantly

Tab. 1. Cohort characteristics, elasticity, shear wave velocity and vascular index values of benign and malignant lesions

\begin{tabular}{|l|c|c|}
\hline & Benign $(\boldsymbol{n}=\mathbf{3 3})$ & Malignant $(\boldsymbol{n}=\mathbf{4})$ \\
\hline Age (mean \pm SD) & $48.6 \pm 16.6$ & $45.0 \pm 30.6$ \\
\hline Gender (F/M) & $11 / 22$ & $1 / 3$ \\
\hline Tumor volume $\left(\mathrm{mm}^{3}\right)$ & $342 \pm 284$ & $401 \pm 359$ \\
\hline Elasticity (in kilopascals) & $47.7 \pm 27.3$ & $55.6 \pm 17.9$ \\
\hline $\begin{array}{l}\text { Shear wave velocity } \\
\text { (in meters per second) }\end{array}$ & $3.6 \pm 1.4$ & $4.1 \pm 0.8$ \\
\hline Relative elasticity & $2.6 \pm 2.8$ & $5.1 \pm 1.4$ \\
\hline $\begin{array}{l}\text { Relative shear wave } \\
\text { velocity }\end{array}$ & $1.4 \pm 0.81$ & $2 \pm 0.4$ \\
\hline Vascular index & $6.2 \pm 7.5$ & $4.7 \pm 4.5$ \\
\hline
\end{tabular}

Tab. 2. Cohort characteristics, elasticity, shear wave velocity and vascularity index values of benign and malignant lesions

\begin{tabular}{|l|c|c|c|}
\hline & $\begin{array}{c}\text { Pleomorphic } \\
\text { adenoma } \\
(\boldsymbol{n}=\mathbf{1 4})\end{array}$ & $\begin{array}{c}\text { Warthin } \\
\text { tumor } \\
(\boldsymbol{n}=\mathbf{1 6})\end{array}$ & p-value \\
\hline Age (mean \pm SD) & $41 \pm 14.1$ & $58.5 \pm 11$ & $\leq 0.001^{*}$ \\
\hline Gender (F/M) & $8 / 6$ & $1 / 15$ & $0.002^{*}$ \\
\hline Tumor volume (mm $\left.{ }^{3}\right)$ & $304 \pm 614$ & $411 \pm 267$ & 0.13 \\
\hline Elasticity (in kilopascals) & $57.5 \pm 31.3$ & $40 \pm 21.3$ & 0.11 \\
\hline $\begin{array}{l}\text { Shear wave velocity } \\
\text { (in meters per second) }\end{array}$ & $4.0 \pm 1.8$ & $3.3 \pm 0.9$ & 0.52 \\
\hline Relative elasticity & $3.2 \pm 2.6$ & $1.5 \pm 0.9$ & 0.64 \\
\hline $\begin{array}{l}\text { Relative shear } \\
\text { wave velocity }\end{array}$ & $1.6 \pm 0.8$ & $1.2 \pm 0.4$ & 0.22 \\
\hline Vascularity index & $2.9 \pm 3.1$ & $9.5 \pm 9.5$ & $0.012^{*}$ \\
\hline
\end{tabular}






Fig. 4. Shear wave elastography (A) and color superb microvascular imaging (B) examinations of a 37-year-old female patient having a pathologically proven Warthin tumor on the left parotid gland. The vascular index of the lesion was 20.6

higher in Warthin tumors than in pleomorphic adenomas $(p=0.012)$. ROC analysis revealed that a cut-off value of 4.05 for the vascular index discriminates pleomorphic adenoma from Warthin tumors with $68 \%$ sensitivity and $72 \%$ specificity (the area under the curve was 0.768 ). Fig. 3, Fig. 4 and Fig. 5 demonstrate the SWE and cSMI ultrasound examinations of a pleomorphic adenoma, Warthin tumor and adenoid cystic carcinoma lesions, respectively.

\section{Discussion}

The parotid gland is the largest salivary gland in the human body. Approximately $80 \%$ of salivary gland tumors arise in the parotid gland, and approximately $80 \%$ of them are benign. The most common tumor of the parotid gland is pleomorphic adenoma ${ }^{(14)}$. Our study group included 33 $(89.1 \%)$ benign lesions, and $4(11.9 \%)$ malignant lesions, and this ratio was slightly higher than the rates reported in the literature. The most common tumor type in this study was Warthin tumor (43.2\%).

Physical examination has an important role in the diagnosis of parotid gland lesions. US has a great advantage in the diagnostic work-up of such lesions because the parotid gland is a superficial organ. However, preoperative characterization of the lesions is usually challenging ${ }^{(14,15)}$. Sonoelastography is an imaging method that evaluates the stiffness and elasticity of tissues. It is particularly useful in the assessment of the

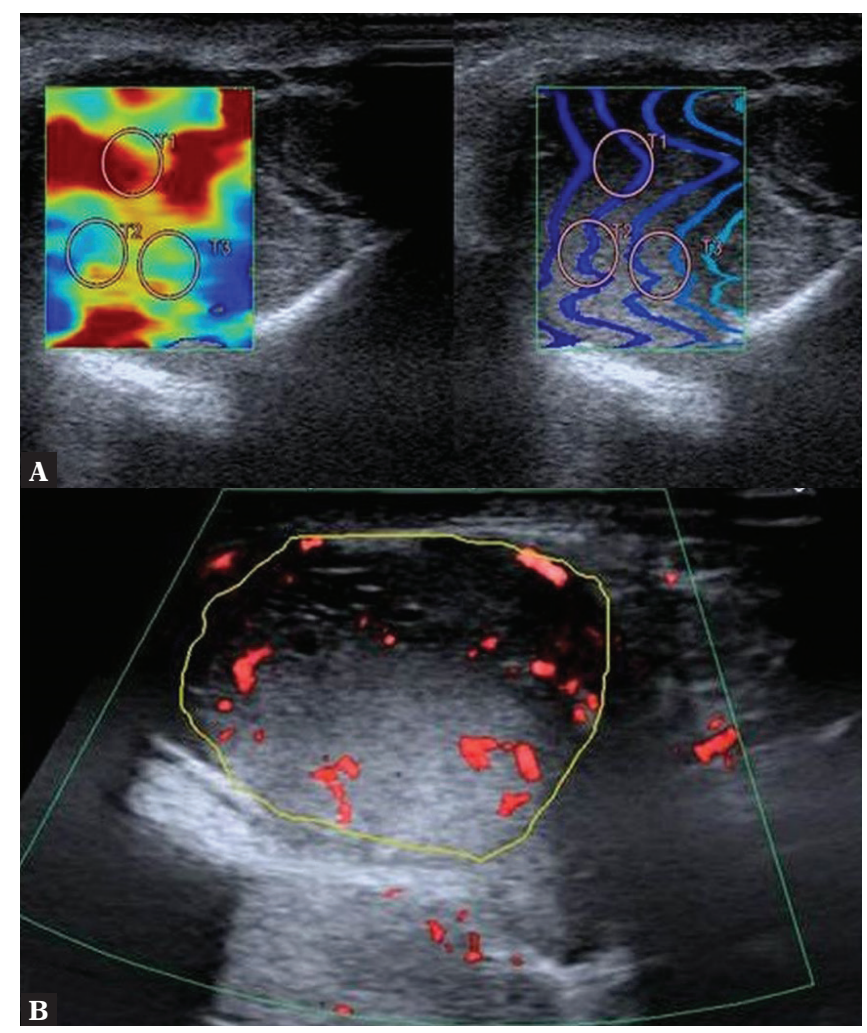

Fig. 5. Shear wave elastography (A) and color superb microvascular imaging (B) examinations of a 22-year-old female patient having a pathologically proven adenoid cystic carcinoma on the right parotid gland. The vascular index of the lesion was 11.2

thyroid, breast and superficial salivary glands ${ }^{(16)}$. According to the results of this study, in which parotid gland lesions were evaluated preoperatively by SWE and SMI, there was no significant difference between benign and malignant lesions evaluated by SWE. In addition, SWE could not distinguish between pleomorphic adenomas and Warthin tumors. There are various studies investigating SWE in the characterization of parotid tumors in the literature (1,2,17-20). $^{(20}$. In a study conducted by Bhatia et al., it was reported that the qualitative elastography score had a poor ability to differentiate between benign and malignant parotid gland lesions ${ }^{(20)}$. Wierzbicka et al. reported a sensitivity of $80 \%$ and specificity of $45.5 \%$ for the elastography score value of $2,60 \%$ and $69.7 \%$ for the elastography score value of 3 , and $40 \%$ and $97 \%$ for the elastography score value of $4^{(18)}$. In a study conducted by Herman et al. in 2017, a total of 97 benign and 28 malignant parotid lesions were evaluated with $\mathrm{SWE}^{(1)}$. The authors found that the maximum stiffness of the ROI alone was a reliable univariate predictor of malignancy ( $\rho=0.0008)$, while the minimum stiffness was a fairly good predictor. Depending on this result, they created a new parameter called the coefficient of stiffness value (CSV) as a ratio of maximum and minimum stiffness values. They concluded that the CSV was a strong predictor of malignancy $(\rho<0.0001)$ which discriminated malignant and benign findings better than any other SWE parameter. The measurement of lesion stiffness in their study was different from our technique. However, our study group included only 4 malignant lesions, and so the statistical comparison 
between the two groups was not possible. Liu et al. reported that the shear wave velocity values of the malignant lesions were higher than those of the benign parotid lesions ${ }^{(21)}$. However, even though their measurement technique of lesion stiffness and elasticity was like in our study, our findings were different from theirs. In another study conducted by Bhatia et al., the authors reported that the pleomorphic adenomas had significantly higher elasticity values than Warthin tumors in their series, inconsistently with our results ${ }^{(20)}$.

In a recent meta-analysis by Zhang et al., a total of 10 studies (711 patients and 725 parotid gland lesions) investigating SWE in parotid tumors were evaluated ${ }^{(22)}$. The authors concluded that sonoelastography had a limited and nonsatisfactory value in the differential diagnosis between malignant and benign parotid lesions. Also, they indicated that quantitative and semi-quantitative SWE methods performed better than qualitative ones. The result of our study is consistent with the findings of this recent meta-analysis.

In the comparison of pleomorphic adenomas and Warthin tumors in terms of age and gender, the male gender was significantly higher in the Warthin tumor group $(\mathrm{M} / \mathrm{F}=$ $15 / 1)$, however no difference was recorded in the pleomorphic adenoma group. The patients with Warthin tumor were older than the patients having pleomorphic adenomas. The results are consistent with previous studies found in the literature $\mathrm{e}^{(23,24)}$.

SMI has been described in the current literature as a novel non-invasive diagnostic imaging technique that enables tissue vessel imaging without using intravenous contrast medium. The SMI technique visualizes low-velocity blood flow in the capillaries with reducing motion artifacts ${ }^{(25)}$. The results of this study show that the vascular index obtained using cSMI is a helpful tool in the presurgical evaluation of parotid gland tumors, particularly for differentiating pleomorphic adenoma and Warthin tumors. To our knowledge, only a few reports can be found in the literature which evaluate the role of SMI in parotid gland tumors. Previous studies with SMI have addressed thyroid gland, lymph nodes, testes, and breast lesions ${ }^{(10,13,25-29)}$.

Previous studies available in the literature which evaluated the vasculature of parotid gland tumors commonly used color and/or power Doppler as the US imaging modality. Those studies commonly found that Warthin tumors were more vascular compared to pleomorphic adenomas ${ }^{(19,30)}$. However, color and power Doppler US exhibit qualitative scores for lesion vascularity. cSMI is more advantageous, as it provides quantitative values. In a recent study by Caliskan et al. ${ }^{(31)}$, the normal quantitative vascular index values of parotid glands in children and adolescents were reported. According to their results, the vascular index was $5 \pm 1.7$ in the right parotid gland, and $4 \pm 1.7$ in the left parotid gland. We did not perform SMI to the normal parotid gland, but we only measured the vascular index of the tumor via drawing the lesion borders. A very recent study conducted by Ryoo et al. ${ }^{(13)}$ in 2018 investigated SMI in pleomorphic adenoma and Warthin tumors of the salivary glands. The authors of that study graded the internal vascularity of salivary gland tumors using a 5-point scoring system. They concluded that the SMI examination increased the level of diagnostic accuracy in the discrimination of pleomorphic adenomas from Warthin tumors. A vascular pattern analysis (vascular distribution and internal vascularity) based on SMI findings together with gray scale sonography can be helpful for distinguishing between pleomorphic adenomas and Warthin tumors. They did not use the quantitative vascular index in their study. One of the advantages of our study is that the quantitative vascular index of lesions obtained by cSMI provided less operator dependence, and a cut-off value was also provided for discriminating pleomorphic adenomas and Warthin tumors.

This study also had some limitations. The first major limitation was the small number of lesions in the malignant tumor group. Only four of 37 parotid lesions were diagnosed as malignant. Another limitation was that some of the lesions were not excised surgically. Pathological diagnoses were made with tru-cut biopsy in 5 lesions, and fine-needle aspiration biopsy in 7 lesions. In some cases, preoperative fine needle or tru-cut biopsy results might differ from the exact histopathological results obtained after surgical excision. From this point of view, if all the patients had undergone surgery, there might be slight differences in the pathological results. To our knowledge, this is the first study evaluating the role of the vascular index obtained by cSMI in the pre-surgical characterization of parotid gland tumors. Although this study was performed in a small number of patients, it is important to note that it is the first such study in the literature. Further prospective studies are needed in larger patient groups.

\section{Conclusion}

SMI is a novel ultrasound technique that is useful in the pre-surgical evaluation of tumors of the parotid gland. The vascular index determined by cSMI can help to differentiate pleomorphic adenomas from Warthin tumors. We could not find a significant difference with SWE between benign and malignant parotid tumors, and also pleomorphic adenomas and Warthin tumors did not reveal a difference with SWE.

\section{Conflict of interest}

Authors do not report any financial or personal connections with other persons or organizations, which might negatively affect the contents of this publication and/or claim authorship rights to this publication. 


\section{References}

1. Heřman J, Sedláčková Z, Vachutka J, Fürst T, Salzman R, Vomáčka J et al.: Differential diagnosis of parotid gland tumors: Role of shear wave elastography. Biomed Res Int 2017; 2017: 9234672.

2. Bhatia KS, Cho CC, Tong CS, Lee YY, Yuen EH, Ahuja AT: Shear wave elastography of focal salivary gland lesions: preliminary experience in a routine head and neck US clinic. Eur Radiol 2012; 22: 957-965.

3. Cantisani V, David E, Sidhu P, Sacconi B, Greco A, Pandolfi F et al.: Parotid gland lesions: multiparametric ultrasound and MRI features. Ultraschall Med 2016; 37: 454-471.

4. Sebag F, Vaillant-Lombard J, Berbis J, Griset V, Henry JF, Petit P et al.: Shear wave elastography: a new ultrasound imaging mode for the differential diagnosis of benign and malignant thyroid nodules. J Clin Endocrinol Metab 2010; 95: 5281-5288.

5. Athanasiou A, Tardivon A, Tanter M, Sigal-Zafrani B, Bercoff J, Deffieux T et al.: Breast lesions: quantitative elastography with supersonic shear imaging - preliminary results. Radiology 2010; 256: 297-303.

6. Evans A, Whelehan P, Thomson K, McLean D, Brauer K, Purdie C et al.: Quantitative shear wave ultrasound elastography: initial experience in solid breast masses. Breast Cancer Res 2010; 12: R104.

7. Tanter M, Bercoff J, Athanasiou A, Deffieux T, Gennisson J-L, Montaldo G et al.: Quantitative assessment of breast lesion viscoelasticity: initial clinical results using supersonic shear imaging. Ultrasound Med Biol 2008; 34 :1373-1386.

8. Kara M, Caliskan E, Atay G, Sutcu M, Kaba O, Adaletli I et al.: Shear wave elastography of parotid glands in pediatric patients with HIV infection. Radiol Med 2019: 124: 126-131.

9. Uysal E, Öztürk M: Quantitative assessment of thyroid glands in healthy children with shear wave elastography. Ultrasound Q 2019; 35: 297-300.

10. Durmaz MS, Sivri M: Comparison of superb micro-vascular imaging (SMI) and conventional Doppler imaging techniques for evaluating testicular blood flow. J Med Ultrason 2018: 45: 443-452.

11. Machado P, Forsberg F: Toshiba Medical System. Medical review initial experience with a novel microvascular flow imaging technique. 2017.

12. Hata J: Seeing the unseen. New techniques in vascular imaging. Superb micro-vascular imaging. Toshiba Med Rev 2014: 1-8.

13. Ryoo I, Suh S, Lee YH, Seo HS, Seol HY, Woo JS et al.: Vascular pattern analysis on microvascular sonography for differentiation of pleomorphic adenomas and warthin tumors of salivary glands. J Ultrasound Med 2018; 37: 613-620.

14. Altinbas NK, Gundogdu Anamurluoglu E, Oz II, Yuce C, Yagci C, Ustuner $\mathrm{E}$ et al.: Real-time sonoelastography of parotid gland tumors. J Ultrasound Med 2017; 36: 77-87.

15. Guzzo M, Locati LD, Prott FJ, Gatta G, McGurk M, Licitra L: Major and minor salivary gland tumors. Crit Rev Oncol Hematol 2010; 74: 134-148.

16. Klintworth N, Mantsopoulos K, Zenk J, Psychogios G, Iro H, Bozzato A: Sonoelastography of parotid gland tumours: initial experience and identification of characteristic patterns. Eur Radiol 2012; 22: 947-956.

17. Olgun DC, Kantarci F, Taskin U, Kilic F, Oktay MF, Altinay S et al.: Relative proportions of stromal to cellular components of pleomorphic adenomas: determination with shear wave elastography. J Ultrasound Med 2014; 33: 503-508.

18. Wierzbicka M, Kałużny J, Szczepanek-Parulska E, Stangierski A, Gurgul E, Kopeć T et al.: Is sonoelastography a helpful method for evaluation of parotid tumors? Eur Arch Otorhinolaryngol 2013; 270: 2101-2107.

19. Mansour N, Stock KF, Chaker A, Bas M, Knopf A: Evaluation of parotid gland lesions with standard ultrasound, color duplex sonography, sonoelastography, and acoustic radiation force impulse imaging - a pilot study. Ultraschall Med 2012; 33: 283-288.

20. Bhatia KS, Rasalkar DD, Lee Y-P, Wong K-T, King AD, Yuen H-Y et al.: Evaluation of real-time qualitative sonoelastography of focal lesions in the parotid and submandibular glands: applications and limitations. Eur Radiol 2010; 20: 1958-1964.

21. Liu G, Wu S, Liang X, Cui X, Zuo D: Shear wave elastography improves specificity of ultrasound for parotid nodules. Ultrasound Q 2018; 34: 62-66.

22. Zhang Y-F, Li H, Wang X-M, Cai Y-F: Sonoelastography for differential diagnosis between malignant and benign parotid lesions: a meta-analysis. Eur Radiol 2019; 29: 725-735.

23. Yoo GH, Eisele DW, Askin FB, Driben JS, Johns ME: Warthin's tumor: A 40-year experience at the johns hopkins hospital. Laryngoscope 1994; 104: 799-803.

24. Pinkston JA, Cole P: Incidence rates of salivary gland tumors: results from a population-based study. Otolaryngol Head Neck Surg 1999; 120: $834-840$.

25. Durmaz MS, Akyürek N, Kara T, Ateş F, Özbakir B, Durmaz FG et al.: Quantitative assessment of thyroid gland vascularization with vascularization index using color superb microvascular imaging in pediatric patients with hashimoto thyroiditis. Ultrasound Q 2019; 35: 281-289.

26. Pei S, Cong S, Zhang B, Liang C, Zhang L, Liu J et al.: Diagnostic value of multimodal ultrasound imaging in differentiating benign and malignant TI-RADS category 4 nodules. Int J Clin Oncol 2019; 24: 632-639.

27. Wakisaka N, Endo K, Kitazawa T, Shimode Y, Kato K, Moriyama-Kita M et al.: Detection of sentinel lymph node using contrast-enhanced agent, Sonazoid $^{\mathrm{TM}}$, and evaluation of its metastasis with superb microvascular imaging in oral and oropharyngeal cancers: a preliminary clinical study. Acta Otolaryngol 2019; 139: 94-99.

28. Liu W, Zhou P, Zhao Y, Tian S, Wu X: Superb microvascular imaging compared with contrast-enhanced ultrasound for assessing laser ablation treatment of benign thyroid nodules. Biomed Res Int 2018; 2018: 1025657.

29. Zhu Y-C, Zhang Y, Deng S-H, Jiang Q, Shi X-R, Feng L-L: Evaluation of plasma cell mastitis with superb microvascular imaging. Clin Hemorheol Microcirc 2019; 72: 129-138.

30. Yuan W-H, Hsu H-C, Chou Y-H, Hsueh H-C, Tseng T-K, Tiu C-M: Grayscale and color Doppler ultrasonographic features of pleomorphic adenoma and Warthin's tumor in major salivary glands. Clin Imaging 2009; 33: 348-353.

31. Caliskan E, Ozturk M, Bayramoglu Z, Comert RG, Adaletli I: Evaluation of parotid glands in healthy children and adolescents using shear wave elastography and superb microvascular imaging. Radiol Med 2018; 123: 710-718. 